\title{
Quantitative Magnetic Resonance Angiography
}

\author{
F. Kellner-Weldon
}

Published online: 4 February 2012

(C) Springer-Verlag 2012

\begin{abstract}
Abbreviations
MCA middle cerebral artery

MRA magnetic resonance angiography

MRI magnetic resonance imaging

NOVA noninvasive optimal vessel analysis

PCMR phase-contrast magnetic resonance

TOF time-of-flight
\end{abstract}

A recently commercially available software, noninvasive optimal vessel analysis (NOVA, VasSol, Chicago, USA), which allows flow quantification by magnetic resonance imaging (MRI), could turn out to be the add-on that Doppler ultrasound was for vascular ultrasound. Quantitative analysis of flow in cerebropetal blood vessels has been of utmost interest for decades. Although phase-contrast magnetic resonance (PCMR) angiography has been available since the early 1980s the practical application in clinical settings was time consuming and not fit for routine [1]. The development of the technique which includes a three dimensional (3D) localizer, as described in the paper by Zhao [2], was the kick-off for what can now be effortlessly applied by neuroradiologists to non-invasively assess the flow patterns in patients with complex disorders, especially as the method is driven by a graphical user interface.

The NOVA software uses magnetic resonance angiography technology (MRA) to quantitatively measure blood flow within the vasculature. The technique is Food and Drug Administration (FDA) approved since 2002 and has

F. Kellner-Weldon $(\square)$

Neuroradiologie, Inselspital, Bern, Switzerland

e-mail: Frauke.Kellner-Weldon@insel.ch a Europe CE Marking Certificate and Iso-certification since 2007.

The experience is based on a test version (7.01) that was installed by a company's collaborator at this site. The test version was available for 3 months and delivered with a head and neck module to be tested in the department for neuroradiology. A significant fee was charged independent of whether the product would be acquired later.

The workflow first consists of acquisition of a MRA-TOF on the MR imager. The scanner used was a 3 Tesla machine but the manufacturer confirmed that 1.5 Tesla machines should perform just as well. The TOF image is sent from the imager to a separate workstation which is linked to the MRimager and has the NOVA software installed.

The 2D-TOF images are cropped in axial, sagittal and coronal planes and fitted to yield a 3D surface-rendering of the vascular anatomy (Fig. 1).

This 3D model (Fig. 2) helps to locate the desired vessel and ensure proper positioning of a slice of interest (cutlocation) which is automatically oriented perpendicularly to the vessel when placed. The quality of the TOF is crucial: if the contrast is too poor this causes difficulties in identifying vessels correctly; moreover, flow data may be incorrect. Perhaps surprisingly it is not possible to place a location-cut in modeled vessels which are not fully contrasted. Most of the time these shortcomings can be overcome by recreating the $3 \mathrm{D}$ model with modifications but if that does not improve the quality repeating the TOF scan may be necessary.

The selected cut-locations are then transferred to the MR-imager for blood flow determination. According to the selected module (this test version came with a head-neck module but body modules are also available) there is a preselected list of abbreviations for the standard vessels available. Cut-locations are defined as "prescriptions" and include 


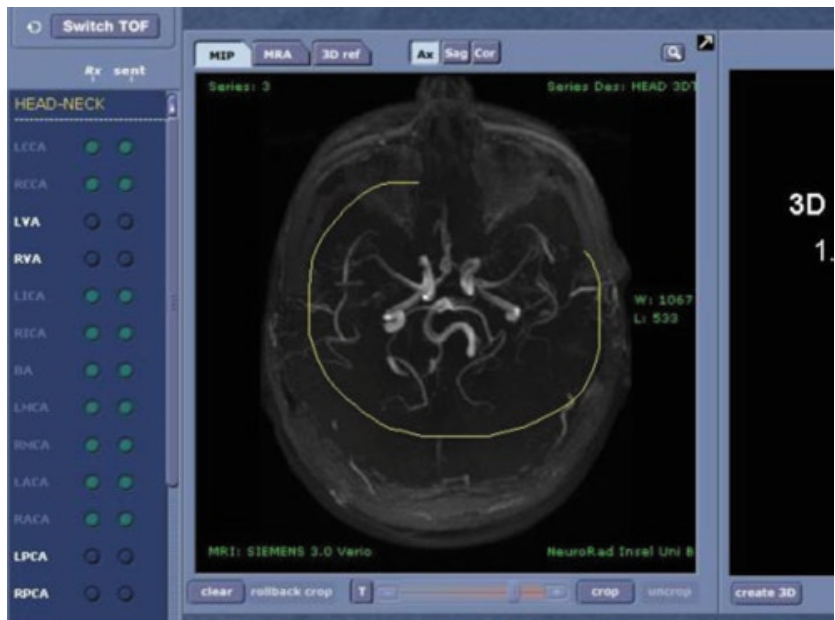

Fig. 1 Example of three dimensional surface-rendering of the vascular anatomy

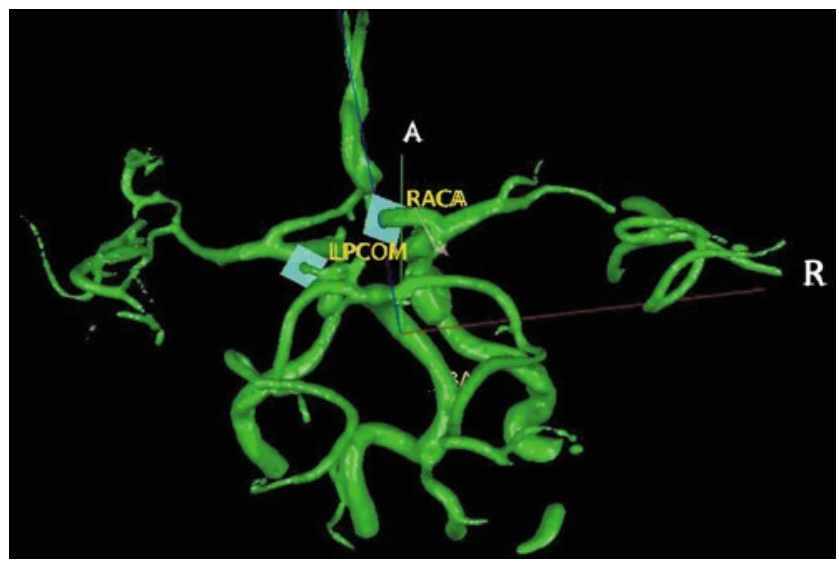

Fig. 2 Resulting three dimensional model of vascular anatomy. $A$ anterior, LPCOM left posterior communicating artery, $R$ right, $R A C A$ right anterior cerebral artery

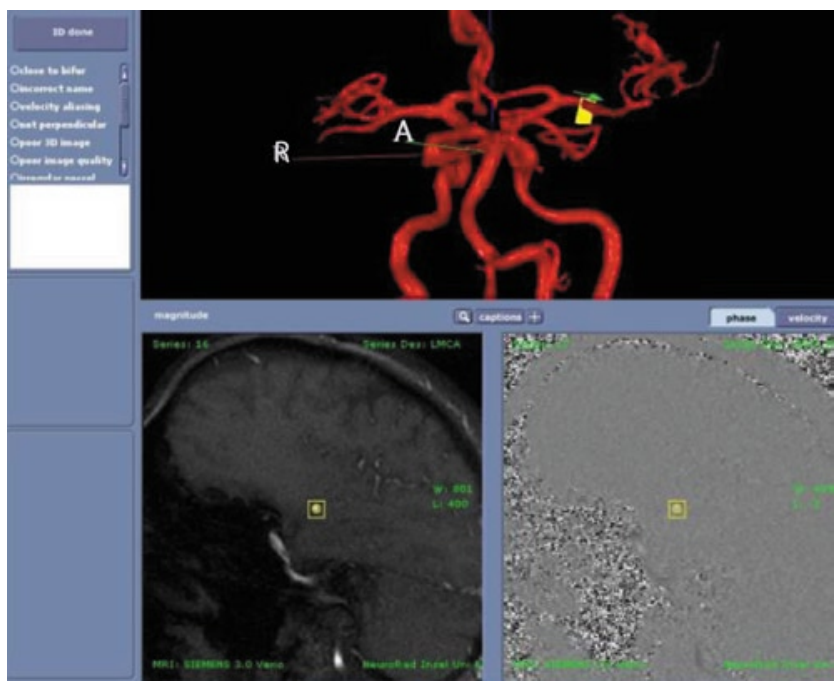

Fig. 3 Identification of the vessel. Magnitude images (lower left), phase images (lower right), model (upper row)

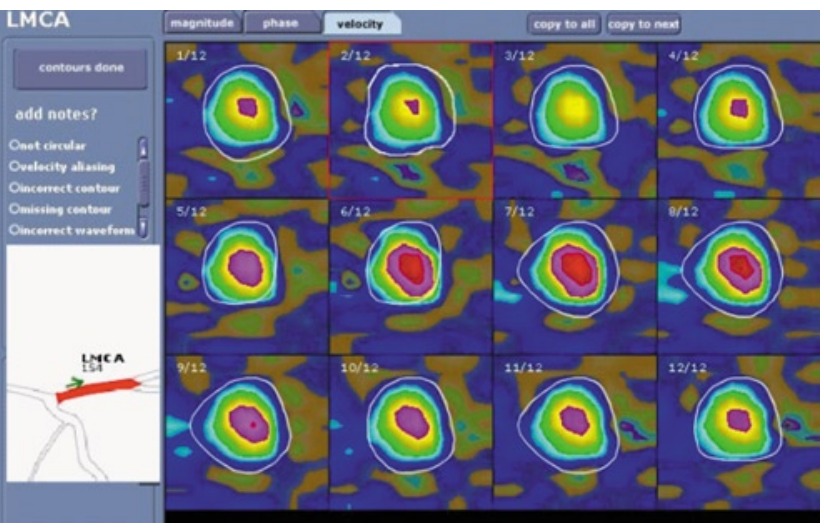

Fig. 4 Color coded velocity images. Velocity highest in center (red), lowest in the periphery (blue). Direction indicated for the flow (left lower image)

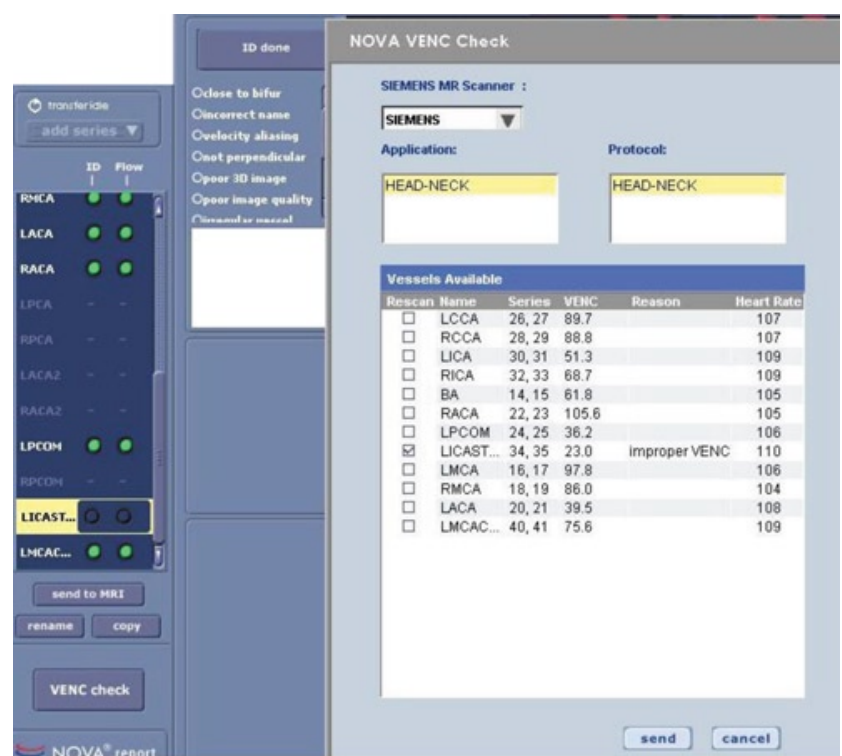

Fig. 5 Venc check flags improper venc estimations and offers rescanning with automated adaptation for velocity encoding

the name or abbreviation of the vessel. Renaming is possible, however, with important caveats (explained later).

The phase-contrast scanning of a vessel cut-location takes $38 \mathrm{~s}$ according to the Siemens protocol and needs pulse gating. Arrhythmia or cold extremities create difficulties in that the scanner breaks off the scanning process due to loss of the pulse signal. While for arrhythmia there is hardly any hope of NOVA measurements, relocating the pulse gate-sensor in case of cold extremities helped most of the time.

The phase-contrast images are forwarded from the scanner to the NOVA workstation to quantify the blood flow.

The flow analysis and quantification procedures come in three steps.

1. The identification of the vessel must be confirmed on the magnitude and phase-contrast image (Fig. 3). 


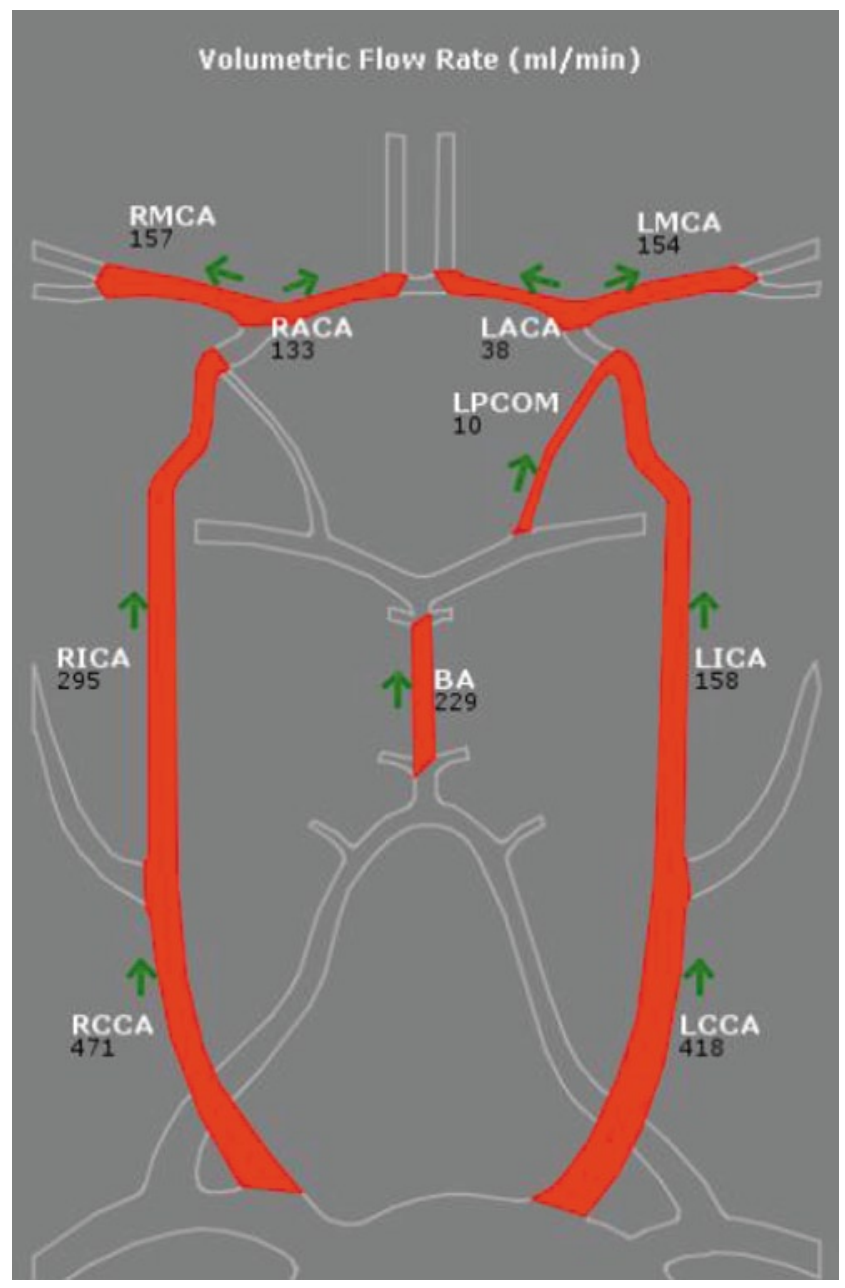

Fig. 6 Schematic diagram of the circulation. Report for a patient with known stenosis of the left carotid artery. The flow in the left MCA drops from $154 \mathrm{ml} / \mathrm{min}$ to $137 \mathrm{ml} / \mathrm{min}$ with inhalative $\mathrm{CO}_{2}$ stimulus which was interpreted as a reduced reserve capacity of the MCA territory. However, the manual naming of the vessel (LMCACO2_2) (see Fig. 7) omits its mapping on the graphical vessel map

2. Color coded velocity images from the magnitude and phase images are provided by the phase-contrast MR studies and the 3D location-cuts. The contour of the vessel must accurately track the vessel border. Experience has shown that the method is robust to minor differences in contours with insignificantly differing flow rates (Fig. 4).

\section{Flow verification}

The system is set up to automatically estimate the venc (velocity encoding) but sometimes needs correction. The correction can be done manually by eliminating aliasing in the contour process and is advised by NOVA if less than one quarter of the images in the series need correction and if the number of pixels needing correction is less than one third of the total number of pixels in the vessel. Otherwise it is recommended to rescan with an automated adjustment.

\begin{tabular}{|lcrrr|}
\hline \multicolumn{1}{|c}{ VESSEL } & FLOW $(\mathbf{m L} / \mathbf{m i n})$ & AGE $\mathbf{1 8}-\mathbf{4 0}$ & AGE $\mathbf{4 1}-\mathbf{6 0} \mathbf{*}^{*}$ & AGE $>=\mathbf{6} \mathbf{*}^{*}$ \\
\hline TCF & - & $\mathbf{7 5 0 - 1 2 7 8}$ & $\mathbf{6 3 7 - 1 2 6 3}$ & $540-1310$ \\
\hline LCCA & 418 & $254-580$ & $233-523$ & $180-562$ \\
\hline RCCA & 471 & $222-590$ & $221-543$ & $172-590$ \\
\hline LICA & 158 & $169-379$ & $135-365$ & $110-352$ \\
\hline RICA & 295 & $147-377$ & $120-362$ & $92-344$ \\
\hline BA & 229 & $101-231$ & $76-224$ & $77-181$ \\
\hline LMCA & 154 & $90-248$ & $96-218$ & $81-201$ \\
\hline RMCA & 157 & $86-230$ & $83-205$ & $67-193$ \\
\hline LACA & 38 & - & - & - \\
\hline RACA & 133 & - & - & - \\
\hline TACA & 171 & $98-274$ & $95-247$ & $83-229$ \\
\hline LPCOM & 10 & - & - & - \\
\hline LICASTENOSE & - & - & - & - \\
\hline LMCACO2_2 & 137 & - & - & - \\
\hline & & & & \\
\hline
\end{tabular}

Fig. 7 Vessel report shows flow for the performed study in $\mathrm{ml} / \mathrm{min}$ (left column) and normative values for different age groups (right three columns). TCF total cranial flow, $L C C A$ left common carotid artery, $R C C A$ right common carotid artery, $L I C A$ left internal carotid artery, $R I C A$ right internal carotid artery, $B A$ basilar artery, $L M C A$ left middle cerebral artery, $R M C A$ right middle cerebral artery, $L A C A$ left anterior cerebral artery, $R A C A$ right left anterior cerebral artery, $T A C A$ both anterior cerebral arteries, $L P C O M$ left posterior communicating artery, LICASTENOSE stenotic left internal carotid artery, LMCACO2_2 left middle cerebral artery while applying $\mathrm{CO} 2$

Experience has shown that corrections are necessary if vessels are very small in diameter (less than $2 \mathrm{~mm}$ ) or have very low flow or oscillating flow and in complex situations when location-cuts were close to bifurcations.

The program offers an optional venc check which flags improper venc values (Fig. 5).

For oscillating flow the venc check will keep complaining of improper venc which is itself diagnostic.

If a cut is placed on a small vessel, such as the posterior communicating artery, flow data may be unreliable due to a partial volume effect. Using a higher in-plane pixel resolution can limit this restriction.

The quantification procedure and validation should be finished before the patient leaves the MR table to make sure all data were collected correctly and no rescan is necessary. Depending on how many vessels are to be examined a routine examination can easily bind the evaluator to the workstation for $30 \mathrm{~min}$. The workflow was set up by NOVA in a way that all of the above described steps are meant to be accomplished by a trained technician. As technicians have different levels of expertise in this department trained doctors do the evaluation.

Once the flow study has been completed (by a technician, as intended by the manufacturer) it is submitted to a reviewer (a doctor, as intended by the manufacturer). Thus two workstations are necessary but as doctors are actually doing the task intended for technicians in this department only a single workstation connected to the scanner would have been more suitable. The review process includes accepting, omitting or correcting vessel flow calculation. Once the report is 
Fig. 8 Snapshots of 3D images displaying the site of the location-cut for Left Middle Cerebral Artery (LMCA, left) and the same artery measured while applying $\mathrm{CO} 2$ (LMCACO2_2, right)
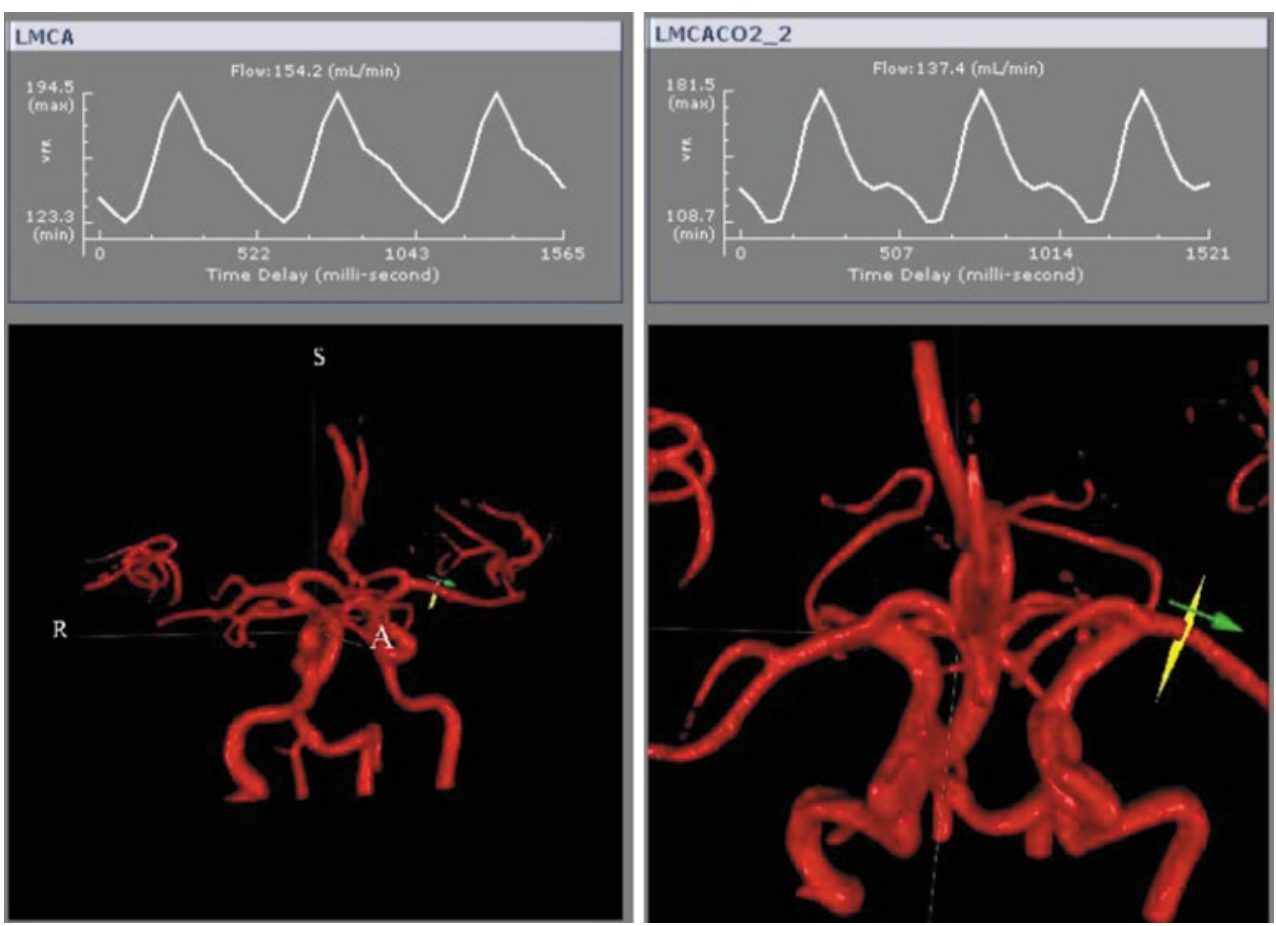

approved the completed study can be pushed forward to a picture archiving and communication system (PACS).

The completed report appears as a browser which includes a schematic diagram of the circulation with flow direction and flow rate values in $\mathrm{ml} / \mathrm{min}$. Any vessel that was named manually will not be depicted on the diagram (Fig. 6) but instead annotated on the bottom of the vessel overview (Fig. 7).

The browser includes a summary which contains the values of all vessels studied (Fig. 7). If both vertebral arteries and common carotid arteries were measured, the list includes the total cranial flow (TCF), which is a nice and easy way to compare preoperative and postoperative flow results and depict hyperperfusion.

Also available are the wave forms for each vessel for the pulsatile flow in a cardiac cycle for 12 time points. Not on the report is the velocity value in $\mathrm{cm} / \mathrm{s}$, which would have been helpful for comparison with Doppler studies.

Snapshots of 3D images indicating the location-cut for each vessel measured are part of the report browser (Fig. 8).

\section{Conclusions}

In the department of neuroradiology the NOVA software was found to be a useful extension of diagnostic MR tools for the evaluation of head and neck vessels. Phase-contrast studies on vessels are now possible within a reasonable time period. The graphic user interface is convenient. A thorough teaching of the staff is crucial because the experience and knowledge of the staff determines the quality of the results and the acquisition time. The method has some technical weaknesses, such as assessing small vessels. These uncertain results might benefit from a validation with Doppler ultrasound. The test version leaves room for improvement with respect to listing velocities in $\mathrm{cm} / \mathrm{s}$. It would also be useful if for each module the predefined list of vessels for analysis could be customized to the institute's interests. The manufacturer was unfortunately not yet able to deliver a module for cerebrospinal fluid (CSF) flow studies but promised to include this in an update. Support for software application and hardware requirements by the manufacturer was prompt and helpful.

\section{References}

1. Bryant DJ, Payne JA, Firmin DN, Longmore DB. Measurement of flow with NMR imaging using a gradient pulse and phase difference technique. Comput Assist Tomogr. 1984;8(4):588-93.

2. Zhao M, Charbel FT, Alperin N, Loth F, Clark ME. Improved phase-contrast flow quantification by three-dimensional vessel localization. Magn Reson Imaging. 2000;18(6):697-706. 